TITLE:

\title{
Rhythmic motion of a droplet under a dc electric field
}

$\operatorname{AUTHOR}(S):$

Hase, Masahiko; Watanabe, Shun N.; Yoshikawa, Kenichi

\section{CITATION:}

Hase, Masahiko ...[et al]. Rhythmic motion of a droplet under a dc electric field. Physical Review E 2006, 74(4): 046301.

ISSUE DATE:

2006-10-06

URL:

http://hdl.handle.net/2433/45691

RIGHT:

Copyright (2006) by the American Physical Society. 
PHYSICAL REVIEW E 74, 046301 (2006)

\title{
Rhythmic motion of a droplet under a dc electric field
}

\author{
Masahiko Hase and Shun N. Watanabe* \\ Department of Physics, Graduate School of Science, Kyoto University, Kitashirakawa-Oiwake-cho, Sakyo-ku, Kyoto 606-8502, Japan \\ Kenichi Yoshikawa ${ }^{\dagger}$ \\ Department of Physics, Graduate School of Science, Kyoto University, Kitashirakawa-Oiwake-cho, Sakyo-ku, Kyoto 606-8502, Japan \\ and "Spatio-Temporal Project," Japan Science and Technology Agency (JST), Japan
}

(Received 31 March 2006; published 6 October 2006)

\begin{abstract}
The effect of a stationary electric field on a water droplet with a diameter of several tens micrometers in oil was examined. Such a droplet exhibits repetitive translational motion between the electrodes in a spontaneous manner. The state diagram of this oscillatory motion was deduced; at $0-20 \mathrm{~V}$ the droplet is fixed at the surface of the electrode, at 20-70 V the droplet exhibits small-amplitude oscillatory motion between the electrodes, and at $70-100 \mathrm{~V}$ the droplet shows large-amplitude periodic motion between the electrodes. The observed rhythmic motion is explained in a semiquantitative manner by using differential equations, which includes the effect of charging the droplet under an electric field. We also found that twin droplets exhibit synchronized rhythmic motion between the electrodes.
\end{abstract}

DOI: 10.1103/PhysRevE.74.046301

PACS number(s): 47.55.D-, 47.55.Kf, 87.80.Fe

In chemistry and chemical engineering, the effect of an electric field on a multiphase system such as an emulsion has attracted increasing attention due to the interesting behaviors that have been observed; e.g., deformation and breakup of a single droplet [1-3], formation of a fiberlike chain of droplets [4], return motion [2], and so on. Active studies on the behavior of multiphase flow under a direct electrostatic field have been performed using numerical simulations and experimental methods. However, the mechanism is still poorly understood due to its complexity, in that the effects of inertia, viscosity, surface deformation, and electrohydrodynamic stresses all interact.

On the other hand, among various kinds of microemulsions, water droplets with a diameter of several tens of microns have recently attracted attention as a convenient model of living cells [5-7]. Active studies on manipulation techniques have been performed using laser tweezer [8] and microfluidics [9], where microdroplets simplify the physical assessment of fluid behavior compared to centimeter-sized droplets because at a micrometer scale the effect of inertia on the behavior of the fluid is less important than those of surface tension and viscosity. To examine the effect of an electric field on microdroplets for the purpose of manipulation, we directly observe the repetitive translational motion of microdroplets between two electrodes under the application of dc voltage, which reflect the presence of limit-cycle oscillation in real space.

A surfactant (DOPC, phospholipid with a neutral head group, purchased from WAKO) was solved in oil (rapeseed oil purchased from NAKARAI) by sonication for 90 minutes and used within 24 hours. To prepare microwater droplets, $2 \mu \mathrm{l}$ water was added to $200 \mu \mathrm{l}$ oil containing $10 \mu \mathrm{M}$ sur-

\footnotetext{
*Electronic address: watanabe@ chem.scphys.kyoto-u.ac.jp; URL: http://www.chem.scphys.kyoto-u.ac.jp/nonnonWWW/watanabe/

${ }^{\dagger}$ Corresponding author. Email address: yoshikaw@scphys.kyotou.ac.jp
}

factant. Droplets with a diameter of several tens of $\mu \mathrm{m}$ were obtained after several seconds of vortex agitation. A single droplet was situated between a pair of gold rods as electrodes with a diameter of $100 \mu \mathrm{m}$ and separated by about $400 \mu \mathrm{m}$, and then dc voltage, $\Psi=0-100 \mathrm{~V}$, was applied, where the droplet is charged at each electrode [part 1 of Fig. 1(a)]. We set the $x$ axis on the coaxial line of the electrodes and the $y$ axis perpendicular to the $x$ axis, where origin is midway between the electrodes [part 2 of Fig. 1(a)]. We used a phasecontrast microscope (Nikon TE-300) equipped with a micromanipulation system (Narishige) and a charge-coupled device (CCD) camera that recorded 30 flames per second.

Figure 1(b) shows microscopic images of a single droplet between the electrodes at $100 \mathrm{~V}$. The droplet exhibits repetitive motion without deformation or breaking up. Figure 1(c) shows a plot of position of the droplet ( $x$ value, upper panel; $y$ value, lower panel). The droplet moves along the coaxial line of the electrodes. This oscillatory motion is also generated regardless of the charge and the type of the surfactant, for example, Tween 20 (neutrally charged surfactant) and DOPG (phospholipid with a negatively charged head group). Small air bubbles are generated within the droplet undergoing rhythmic motion, which indicates that the droplet is charged by the electrolysis of water. The gradient of the applied electric field is greatest along the line between the tips of the electrodes, indicating that attractive force on the droplet as a dielectric body acts in the direction of $y=0$.

When the voltage is well below $20 \mathrm{~V}$, a droplet is pulled toward either electrode and is attached to the electrode surface, as exemplified in part 1 of Fig. 2(a) (10 V). At high electrical potentials, a "small oscillation" of a droplet is generated between the origin and an electrode, as exemplified in part 2 of Fig. 2(a) $(50 \mathrm{~V})$, while small oscillation is observed around each electrode. When the voltage exceeds $70 \mathrm{~V}$, "large oscillation" is generated between the electrodes, where the droplet touches both electrodes, as shown in part 3 of Fig. 2(a) $(100 \mathrm{~V})$. The trajectory of droplet motion is on the coaxial line between the electrodes in both small and large oscillations. 
(a)

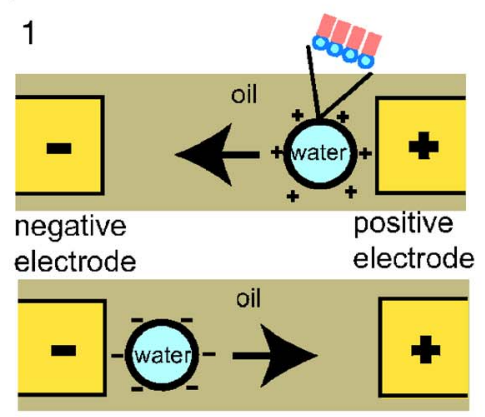

2

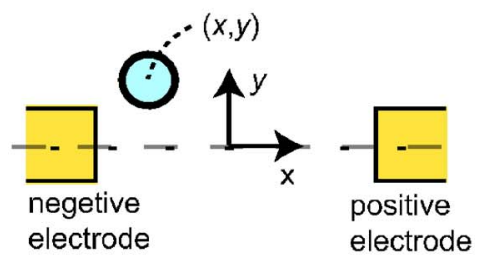

(b)

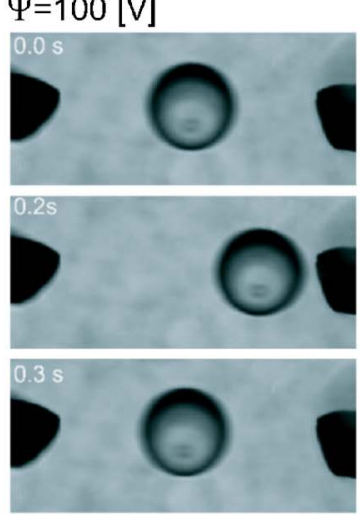

0.55

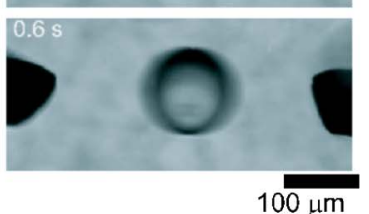

(c)
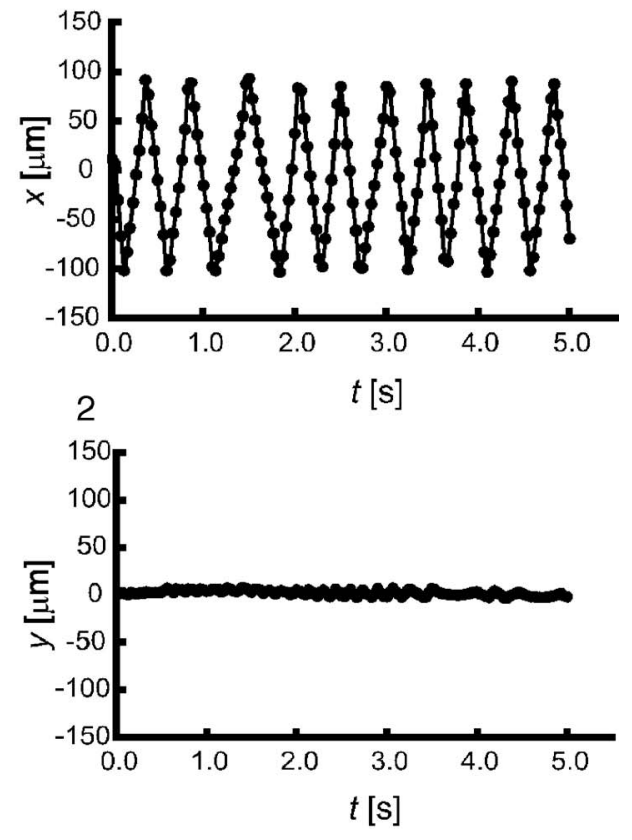

FIG. 1. (Color online) (a) Schematic representation of the effect of a dc electric field on a droplet (part 1) and on the geometrical arrangement (part 2). (b) Repetitive translational motion of a water droplet at $100 \mathrm{~V}$. (c) Time traces under $100 \mathrm{~V}$. (part 1 ) $x$ coordinate. (part 2) $y$ coordinate.

At a micrometer scale, viscosity and surface tension should strongly influence fluid behavior. To evaluate the most important effect under the conditions in the study, the capillary number $C_{a}$, for comparing viscosity and surface tension and the Reynolds number $R_{e}$, for comparing inertia and viscosity, are written as

$$
C_{a}=\frac{\eta v}{\gamma} \sim 10^{-2}
$$

$$
R_{e}=\frac{\rho v r}{\eta} \sim 10^{-4}
$$

where $\eta$ is the viscosity of the oil $\left(\approx 8.4 \times 10^{2} \mathrm{~Pa} \mathrm{~s}\right), v$ is the translational velocity of a droplet $\left(\sim 10^{-3} \mathrm{~m} / \mathrm{s}\right), \gamma$ is the surface tension between water and oil $\left(\sim 10^{-2} \mathrm{~N} / \mathrm{m}\right), \rho$ is the density of the oil $\left(\approx 0.91 \times 10^{3} \mathrm{~kg} / \mathrm{m}^{3}\right)$, and $r$ is the size of a droplet $\left(\sim 10^{-5} \mathrm{~m}\right)$. Next, the electric capillary number $C_{e}$, (a)

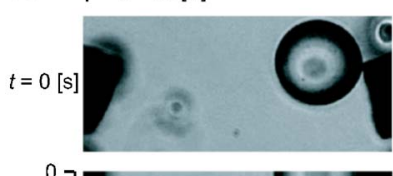

${ }_{2}$

(b)
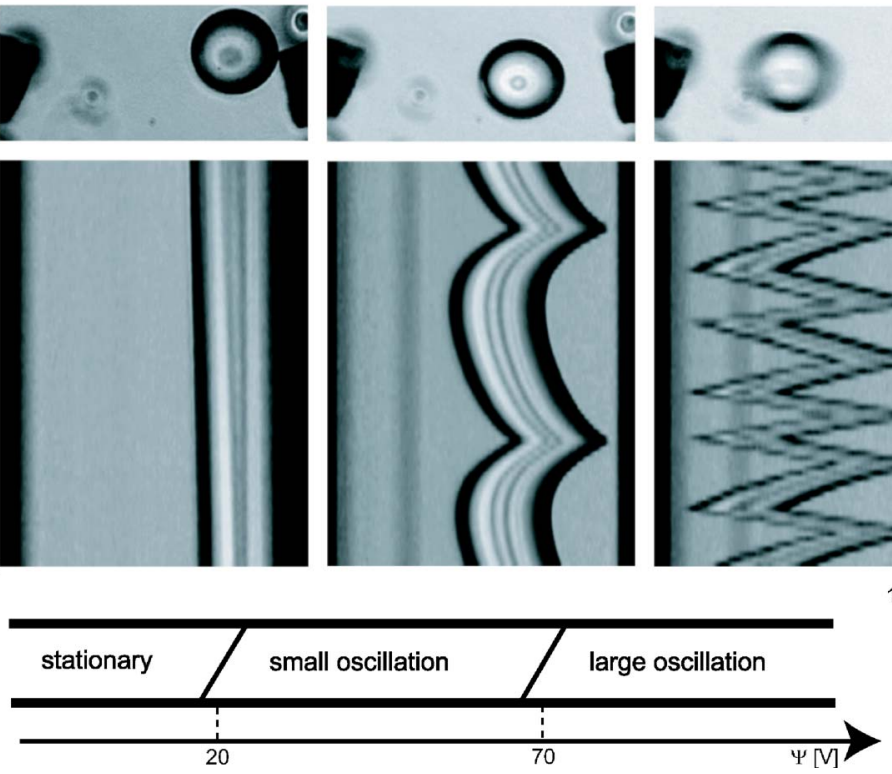

FIG. 2. (Color online) (a) Spatio-temporal pictures of the motion of a droplet at 10, 50, and $100 \mathrm{~V}$, respectively. (b) Phase diagram of the oscillatory motion of a droplet as a function of the applied voltage. 
(a)
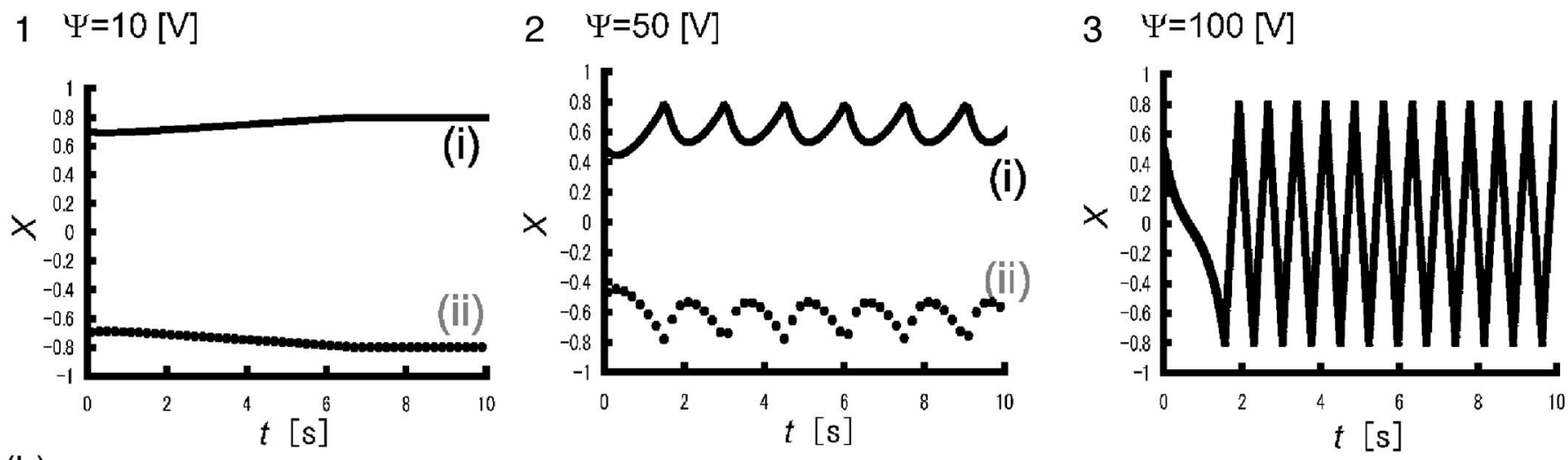

(b)

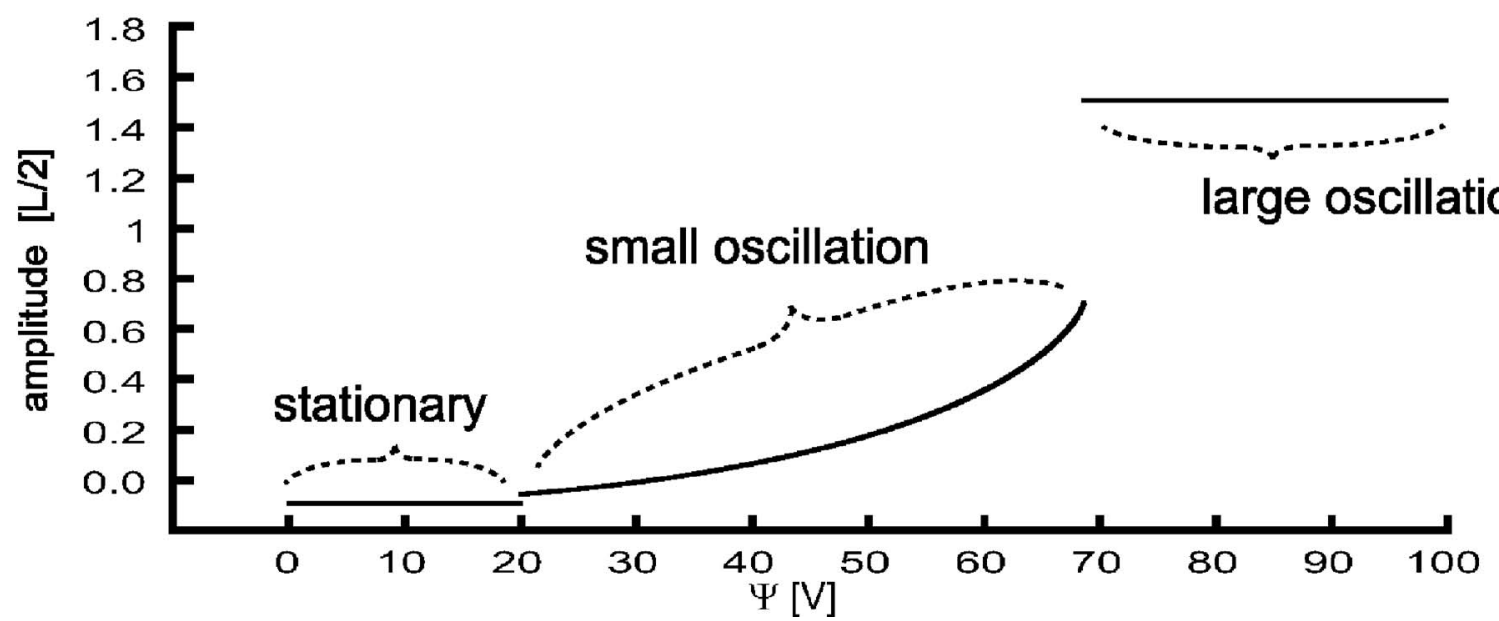

FIG. 3. (a) Numerical simulation of the motion of a droplet under a de electric field. (part 1) $\Psi=10 \mathrm{~V}$ with an initial condition symbolized as (i) and (ii), respectively. (part 2) $\Psi=50 \mathrm{~V}$ with an initial condition symbolized as (i) and (ii), respectively. (part 3) $\Psi$ $=100 \mathrm{~V}$. (b) A plot of amplitude of the oscillatory motion of a droplet at different voltages.

for comparing electrostatic forces and surface tension, is written as

$$
C_{e}=\frac{\epsilon_{w} \epsilon_{o} E^{2} r}{\gamma} \sim 10^{-1},
$$

where $\epsilon_{w}$ is the dielectric constant of water, $\epsilon_{o}$ is the permittivity of vacuum, $E$ is the electrostatic field around a droplet ( $E \approx \Psi / L, L$ is the distance between the electrodes). These considerations suggest that the deformation of a droplet from a spherical shape is negligible and that the effect of inertia on motion is also minimal. In the stationary state, the droplet wets an electrode. It is expected that a droplet would move away from an electrode when repulsive electrostatic force overcomes the attraction of the droplet to the surface of the electrode due to the surface tension, which is written as $\gamma l$ $<q E$, where $l$ is the circumference of the wetting area and $q$ is the charge of the droplet.

The dynamic equation for the position of a droplet can be written as

$$
k \dot{x}=q E-\nabla U(x),
$$

where we ignored the effect of inertia, $k$ is a coefficient of resistance $(k=6 \pi \eta r)$, and $U(x)$ is potential. In the experi- ment, an uncharged droplet is attracted to the nearest electrode regardless of whether the voltage is positive or negative. By considering the attractive force between a droplet and each electrode due to the induced charge of the droplet, by symmetry, $U(x)$ can be written as $U(x)=a \Psi^{2} x^{2}$, where $a$ is a negative constant, we consider that the potential due to the induced charge is proportional to $\Psi^{2}$ and we ignore terms of more than third order of $x$ for simplicity. By taking the effect of charge leakage into consideration, the timedependent change in the charge may be given as

$$
\dot{q}=-\alpha q,
$$

where $\alpha$ is a positive constant. We consider that a droplet obtains a charge $q= \pm C \Psi$ when in contact with an electrode $\left[C=\pi^{3} r^{2} \epsilon \epsilon_{0} / 3 L, C\right.$ is capacitance, where $\epsilon$ is the dielectric constant of oil $(\approx 5)[10]]$. We set new parameters $X$ and $Q$ as $x=\frac{L}{2} X$ and $q=C Q$. With a linear stability analysis, we found that Eqs. (4) and (5) have an unstable solution, $(X, Q)=(0,0)$. For simplicity $a, \alpha, r, L$, and $l$ are set as $-\frac{2 a}{k}=2.5 \times 10^{4}, \alpha=1, r=4 \times 10^{-4} \mathrm{~m}, L=4 \times 10^{-4} \mathrm{~m}$, and $l$ $=2 \times 10^{-7} \mathrm{~m}$. Part 1 of Fig. 3(a) shows the results of a simulation at $10 \mathrm{~V}$, where $(X, Q)=( \pm 0.7, \pm 10)$ at $t=0$. After a droplet touches each electrode, it remains stationary at the 
electrode. Part 2 of Fig. 3(a) shows the results of a simulation at $50 \mathrm{~V}$, where $(X, Q)=( \pm 0.5, \pm 20)$ at $t=0$. A droplet exhibits periodic motion around each electrode with small amplitude, and two limit cycles exist. Part 3 of Fig. 3(a) shows the results of a simulation at $100 \mathrm{~V}$, where $(X, Q)$ $=(0.5,40)$ at $t=0$. A droplet exhibits stable oscillatory motion between the electrodes and touches both electrodes with large amplitude. Figure 3(b) schematically summarizes the mode bifurcation with respect to the applied voltage. A droplet exhibits a stationary mode below $20 \mathrm{~V}$ and two types of modes of repetitive motion, small and large oscillations, exist at $20-70 \mathrm{~V}$ and $70-100 \mathrm{~V}$, respectively, where in the transition between the modes the amplitude changes in a discrete manner and the transition between small and large oscillations occurs with spontaneous spatial symmetry breaking.

It is noted that the numerical result in Fig. 3 reproduces the experimental trend in Fig. 2, indicating that the observed phenomenon in the present study is interpreted in terms of "nonlinear dynamics." As far as we know, there has been no report on the occurrence of periodic translational motion in a solution under dc electric field as a representation of limitcycle oscillation, in spite of the long standing research history in electrochemistry. Since the oscillation exhibits significant nonlinearity, we can expect the generation of rich variety of spatio-temporal modes on the microdroplet under dc field. As an experimental example toward such research direction, we show the synchronized motion of a couple of droplets in Fig. 4. Where the twin droplets simultaneously exhibit rhythmic motion under the synchronized mode of in phase with different amplitude, along the coaxial line between the electrodes at $100 \mathrm{~V}$. We noted that droplets scarcely fuse together. The droplets convey electrons by engaging in rhythmic motion between the electrodes and by exchanging the charge when they touch each other, where the charge exchanged is determined in accordance with the Laplace equation with boundary conditions. The orbits of the droplets are confined to the coaxial line for the same reason as in the case of a single droplet. In addition, we have observed that more than two droplets, to a tested maximum of five droplets, exhibit synchronized rhythmic motion in a similar fashion.

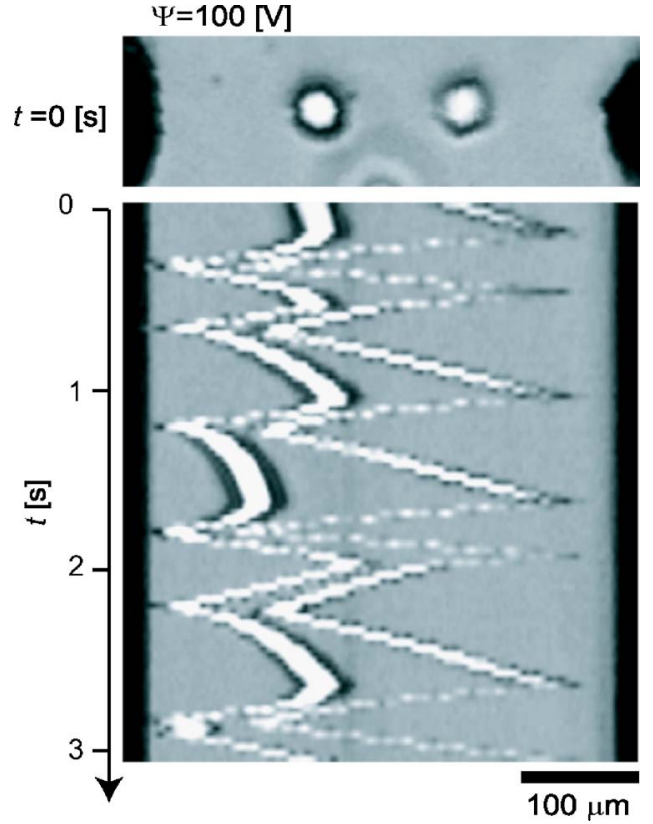

FIG. 4. (Color online) Spatio-temporal pictures of the synchronized rhythmic motion of twin droplets without coalescence at $100 \mathrm{~V}$.

In the present study we showed that periodic oscillatory motion is generated for microwater droplets in oil under a dc electric field. As we have adapted the experimented condition of large interfacial tension, essentially no deformation of the droplet was observed. As a future research subject, it may be of interest to perform the experiment on droplets under low interfacial tension, for example, by adding a surfactant. One such experiment, time-dependent large deformation of the droplet shape may be generated coupled with the translational motion under dc field.

This work was partly supported by a Grant-in-Aid for Scientific Research on Priority Areas (No. 17076007) "System Cell Engineering by Multi-scale Manipulation" from the Ministry of Education, Culture, Sports, Science and Technology of Japan.
[1] G. I. Taylor, Proc. R. Soc. London, Ser. A 291, 159 (1966).

[2] J. S. Eow, M. Ghadiri, and A. Sharif, Colloids Surf., A 225, 193 (2003).

[3] A. Fernández, G. Tryggvason, J. Che, and S. Ceccio, Phys. Fluids 17, 093302 (2005).

[4] W. M. Winslow, J. Appl. Phys. 20, 1137 (1949).

[5] A. V. Pietrini and P. L. Luisi, ChemBioChem 5, 1055 (2004).

[6] D. S. Tawfik and A. D. Griffiths, Nat. Biotechnol. 16, 652
(1998).

[7] M. Hase and K. Yoshikawa, J. Chem. Phys. 124, 104903 (2006).

[8] S. Katsura, A. Yamaguchi, H. Inami, S. Matsuura, K. Hirano, and A. Mizuno, Electrophoresis 22, 289 (2001).

[9] J. Atencia and D. J. Beebe, Nature (London) 437, 648 (2005).

[10] A. Y. H. Cho, J. Appl. Phys. 35, 2561 (1964). 